\title{
Research on the Volatility of China's Stock Market Based on MCMC Algorithm
}

\author{
Yu Wang1, a, Hao Wang', b \\ ${ }^{1}$ School of Science, Beijing Jiaotong University, Beijing 100044, China; \\ ${ }^{2}$ School of Science, Beijing Jiaotong University, Beijing 100044, China. \\ a15271053@bjtu.edu.cn, b15271173@bjtu.edu.cn,
}

Keywords: volatility analysis; MCMC algorithm; SV model.

\begin{abstract}
Financial time series contains information on market changes and investment risk fluctuations. This paper aims to study the volatility of China's stock market by analyzing the time series of Chinese stock market returns. We choose the closing price of the Shanghai and Shenzhen close index as a sample. To analyze the statistical characteristics of the time series, we choose the SVMN model in the SV model family by MCMC algorithm based on Gibbs sampling to estimate the basic characteristics of the stock market volatility. Through research, we find that China's stock market has the characteristics of agglomeration, volatility persistence and Leptokurtosis. We analyze these characteristics and specific market reasons to provide financial and securities service industries with relevant recommendations to stabilizing the market.
\end{abstract}

\section{Introduction}

The purpose of this paper is to describe and study the characteristics of China's stock market volatility by stochastic volatility model that characterizes the volatility characteristics. This paper empirically analyzes the characteristics of stock market volatility and factors that affect volatility to provide theoretical support and decision-making reference in risk management field for investors.

This paper uses SV-MN model to analyze the stock market return rate. Compared with the traditional SV-N model, the SV-MN model can well represent the characteristics of the time series distribution function and is the most suitable model for estimating the leptokurtosis time series in the SV model family. When estimating the model, the Markov Chain Monte Carlo Simulation (MCMC) method has a unique advantage in estimating the SV model and it can simultaneously estimate unknown parameters and unobservable variables.

Through volatility analysis, we find that China's stock market has the characteristics of clustering, sustainability, and time-varying characteristics. We analyze the causes of the stock market volatility in terms of related policies, investor quality, investment psychology, and manipulation behavior to provide the market financial services industry with relevant recommendations for stabilizing the market.

\section{Statistical Characteristics of China's Stock Market}

Firstly, in order to select the appropriate SV model to further analyze the time series of Chinese stocks, we first analyzed the characteristics of the time series of Chinese stock market returns using Shanghai and Shenzhen stock indexes. We chose the Shanghai and Shenzhen stock indices of 700 trading days from January 5, 2015 to November 15, 2017 to calculate this experiment data and eliminate some unnecessary interference factors. The data comes from Flush Software.The financial time rate of return sequence uses the following formula:

$$
y_{t}=\left(\ln p_{t}-\ln p_{t-1}\right)-\frac{1}{n} \sum_{i=1}^{n}\left(\ln p_{i}-\ln p_{i-1}\right)
$$

To measure the volatility and investment risk, we calculate the standard deviation, skewness, kurtosis and autocorrelation coefficient of the lag i-order. By calculation we get the following result: 
TABLE 1 HS INDEX BASIC INDICATORS

\begin{tabular}{|c|c|c|c|}
\hline Standard deviation & Skewness & Kurtosis & Mean value \\
\hline 0.017262 & -1.27474 & 6.675998 & -0.00611 \\
\hline & & & \\
\hline
\end{tabular}

Fig. 1 HS Index Returns Histogram

As can be seen from the results, the skewness of the Shanghai-Shenzhen index is negative, indicating that the mean of the distribution of returns is stronger on the left than on the right, that is, the distribution of returns has a skewed character. The kurtosis is greater than 3 , which shows that the distribution of returns in China's stock market has more obvious characteristics of leptokurtosis than normal distribution. In order to further verify the leptokurtosis nature of the stock time series, we use the following graphical techniques for analysis.

From the Fig.1, it can be seen that the kurtosis of the actual data of the Shanghai-Shenzhen index is higher than the kurtosis of the normal distribution data, the waist is thinner, the tail is thicker, and the histogram is not completely symmetrical, but slightly skewed. Therefore, the leptokurtosis characteristics of China's stock market are again verified.

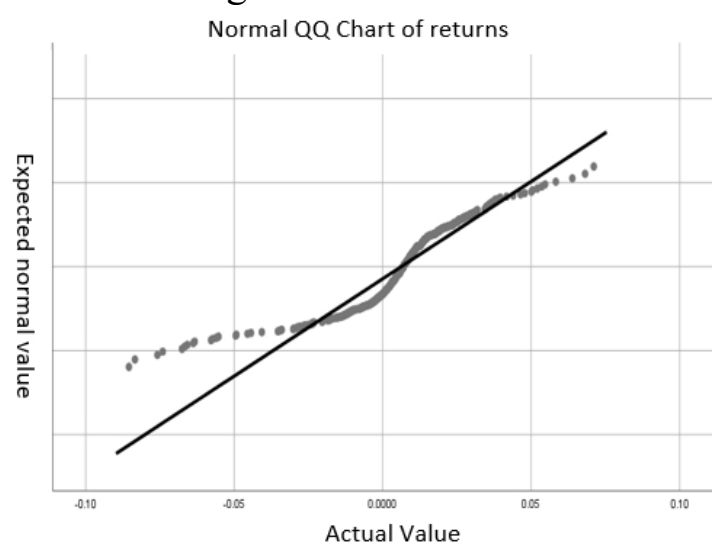

Fig. 2 HS Index Yield QQ Chart

Then we use the QQ graph for analysis. It can be seen from Fig.2 that the return rate of the Shanghai-Shenzhen index is not a straight line, indicating that the distribution of the outliers has a higher probability of being outlier than the normal distribution, that is, the Shanghai and Shenzhen index's return volatility has a clear leptokurtosis feature.

MCMC algorithm. MCMC is a Monte Carlo method that is simulated by a computer under the Bayesian framework. It provides a method for sampling from the posterior distribution of the parameters to be estimated, which allows us to obtain an estimate of the parameters to be estimated or their function values and their distribution. Essentially, the MCMC method is a Monte Carlo integral using a Markov chain. The MCMC method based on the Bayesian inference principle is mainly used to generate posterior distribution samples, calculate the edge distribution and the moment of the posterior distribution.

The basic idea of the MCMC method is to construct a Markov chain with specified stationary distribution to simulate the unknown variable $U_{k}$, and then to iterate the chain a sufficient number of times, when the chain reaches a steady state distribution, the required posterior distribution is obtained. 
The random point $U_{k}$ comes from the distribution $\pi(\mathrm{U})$. Different MCMC methods are obtained by different sampling methods, such as Metropolis-Hastings method, Gibbs sampling method, and various compound methods.

Gibbs sampling. Gibbs sampling is the simplest and most widely used sampling method. Let $X=($ $X_{1}, X, \ldots, X_{n}$ ) be a random variable in $R^{d}$, and the joint distribution $\mathrm{f}(\mathrm{x})$ is the target sampling distribution. Define the d-1 random variable as:

$$
X_{-j}=\left(X_{1}, \ldots, X_{j-1}, X_{j+1} \ldots, X_{d}\right)
$$

Note the conditional density of $X_{j} \mid X_{-j}$ is $\mathrm{f}\left(X_{j} \mid X_{-j}\right), \mathrm{j}=1,2, \ldots, \mathrm{d}$. The sampling method is to generate candidate points from the $\mathrm{d}$ conditional distributions to solve the difficulty of sampling directly from $\mathrm{f}$. The algorithm is as follows:

(a) Initialize $\mathrm{X}(0)$ at $\mathrm{t}=0$;

(b) $\mathrm{t}=1,2, \ldots, \mathrm{T}$;

(1)Let $\mathrm{x}=\mathrm{X}(\mathrm{t}-1)$;

(2)For each component $\mathrm{j}=1,2, \ldots, \mathrm{d}$, generate candidate points $X_{j}{ }^{*}(t)$ from $\mathrm{f}\left(X_{j} \mid x_{-j}\right)$, and then update $x_{j}=X_{j}^{*}(t)$;

(3) Let $\mathrm{X}(\mathrm{t})=\left(X_{1}{ }^{*}(t), \ldots, X_{d}{ }^{*}(t)(\right.$ each candidate points are accepted);

(4) Increase of $t$;

In step (2) above, each component is updated sequentially:

$$
\begin{aligned}
& x_{1}(t) \sim f\left(x_{1} \mid x_{2}(t-1), \ldots, x_{d}(t-1)\right), \\
& x_{2}(t) \sim f\left(x_{2} \mid x_{1}(t), x_{3}(t-1), \ldots, x_{d}(t-1)\right) \\
& \ldots ., \\
& x_{d}(t) \sim f\left(x_{d} \mid x_{1}(t) \ldots, x_{d-1}(t)\right) .
\end{aligned}
$$

Sampling from a univariate distribution $\mathrm{f}\left(x_{j} \mid x_{1}(t), x_{2}(t), \ldots, x_{j-1}(t), x_{j+1}(t-1) \ldots, x_{d}(t-1)\right.$ is relatively easy because $\mathrm{f}\left(x_{j} \mid x_{-j}\right) \propto f(x)$. In addition to $x_{j}$, all other variables are constants. At the same time, under positive conditions, the joint density satisfies the following formula:

$$
f\left(x_{1}, \ldots, x_{d}\right) \propto \prod_{j=1}^{d} \frac{f\left(x_{j} \mid x_{1}, \ldots, x_{j-1}, x_{j+1} \ldots, x_{d}^{\prime}\right)}{f\left(x_{j}^{\prime} \mid x_{1}, \ldots, x_{j-1}, x_{j+1} \ldots, x_{d}^{\prime}\right)},
$$

Therefore, Gibbs sampling method can be used to generate an irreducible Markov chain.

SV-MN model. Through the statistical characteristics analysis in the previous article, we have obtained that the time series of the volatility of China's stock market returns has the characteristics of leptokurtosis. Based on the characteristics of China's stock market, we use the SV-MN model in discrete stochastic volatility model to simulate the volatility of China's stock market. In the model, we make the following assumptions:

\section{TABLE 2 MCMC-SV-MN MODEL PARAMETERS}

\begin{tabular}{|l|l|}
\hline Symbol & Meaning \\
\hline$\mu$ & Fluctuation level parameter \\
\hline$\theta_{t}$ & Volatility persistence parameter \\
\hline$\tau$ & Accuracy Parameters of Shanghai and Shenzhen Index Model \\
\hline $\mathrm{d}$ & Index of correlation between yield and volatility \\
\hline
\end{tabular}

The ARCH-M model proposed by Engle, Lilien, and Robins considers the role of risk compensation in the market and is extended by Koopman to the SV model and proposes the SV-M model:

$$
\begin{gathered}
y_{t}=u_{t}+\varepsilon_{t} \exp \left(\frac{\theta_{t}}{2}\right) \\
\theta_{t}=\mu+\emptyset\left(\theta_{t-1}-\mu\right)+\eta_{t}, \eta_{t} \sim i . i . d N\left(0, \tau^{-1}\right) \\
u_{t}=\alpha+y_{t-1}+\operatorname{dexp}\left(\theta_{t}\right)
\end{gathered}
$$

In the later application, SV-MN model is simplified. 


$$
\begin{aligned}
& y_{t}=\operatorname{dexp}\left(\theta_{t}\right)+\varepsilon_{t} \exp \left(\frac{\theta_{t}}{2}\right), \varepsilon_{t} i . i . d N(0,1) \\
& \theta_{t}=\mu+\emptyset\left(\theta_{t-1}-\mu\right)+\eta_{t^{\prime}} \eta_{t} \sim i . i . d N\left(0, \tau^{-1}\right)
\end{aligned}
$$

Among them, $\operatorname{dexp}\left(\theta_{t}\right)$ is the risk compensation and $\mathrm{d}$ is the regression coefficient of the measured mean fluctuation effect. $\theta_{t}$ obeys the normal distribution with a mean of $\varnothing\left(\theta_{t-1}-\mu\right)$ and a variance of $\tau^{-1}$,which is:

$$
\theta_{t} \mid \mu, \emptyset, \theta_{t-1} \sim \mathrm{N}\left(\mu+\varnothing\left(\theta_{t-1}-\mu\right) \tau^{-1}\right), \mathrm{t}=1,2, \ldots, \mathrm{n}
$$

For a given $\theta_{t}$ and $\mathrm{d}$,

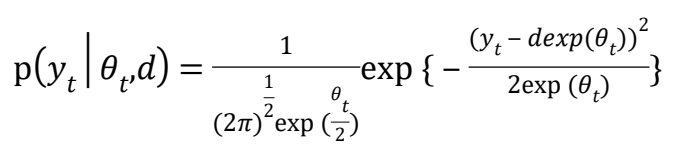

At this time, $y_{t} \mid \theta_{t}, d$ obeys the normal distribution with expectation of $\operatorname{dexp}\left(\theta_{t}\right)$ and variance of $\exp \left(\theta_{t}\right)$, which is

$$
y_{t} \mid \theta_{t}, d \sim N\left(\operatorname{dexp}\left(\theta_{t}\right), \exp \left(\theta_{t}\right)\right)
$$

So the likelihood function of SV-MN is:

$$
\mathrm{L}\left(\mu, \phi, \sigma, d, \theta_{0: n}\right)=\prod_{t=1}^{n} \mathrm{p}\left(y_{t} \mid \theta_{t^{\prime}} d\right)=\prod_{t=1}^{n} \frac{1}{(2 \pi)^{\frac{1}{2}} \exp \left(\frac{\theta_{t}}{2}\right)} \exp \left\{-\frac{\left(y_{t}-\operatorname{dexp}\left(\theta_{t}\right)\right)^{2}}{2 \exp \left(\theta_{t}\right)}\right\}=(2 \pi)^{\frac{n}{2}} \exp \left\{-\frac{1}{2} \sum_{t=1}^{n}\left(\theta_{t}+\frac{\left(y_{t}-\operatorname{dexp}\left(\theta_{t}\right)\right)^{2}}{2 \exp \left(\theta_{t}\right)}\right)\right\}
$$

The prior distribution of $\mu, d, \varnothing, \tau$ is:

$\emptyset \sim B e(20,1.5)$,

$\tau \sim G a(2.5,0.025)$,

$\mu \sim N(0,0.01)$.

\section{Simulation analysis}

We collected the HS300 closing index from January 4, 2010 to December 29, 2017, and processed the improved daily return rate using the following formula

$$
\mathrm{y}_{t}=\left(\ln p_{t}-\ln p_{t-1}\right)-\frac{1}{n} \sum_{i=1}^{n}\left(\ln p_{i}-\ln p_{i-1}\right)
$$

This paper uses openbugs software to simulate the volatility of China's stock market using the SVMN model based on the MCMC algorithm. First, we perform 10,000 iterations of each parameter in the model and then perform annealing to ensure the convergence of the parameters. Then discard the original iteration and proceed with 40,000 iterations. The following figure shows the Bayesian estimates of the mean, standard deviation, $\mathrm{MC}$ error, $2.5 \%$, and $97.5 \%$ quantile of the Shanghai and Shenzhen indices based on the SV-MN model.

$\begin{array}{lllllll} & \text { mean } & \text { sd } & \text { MC_error val2.5pc } & \text { median } & \text { val97.5pc } \\ \text { d } & 0.05861 & 0.6856 & 0.00143 & -1.38 & 0.002645 & 1.544 \\ \text { itau2 } & 58.81 & 49.49 & 0.9011 & 1.675 & 43.33 & 190.1 \\ \text { mu } & -6.349 & 4.316 & 0.07869 & -9.179 & -8.567 & 9.422 \\ \text { phi1 } & 0.9626 & 0.05389 & 9.753 \mathrm{E}-4 & 0.82 & 0.9836 & 0.9967\end{array}$

Fig. 3 Bayesian Parameter Estimation under SV-MN Model
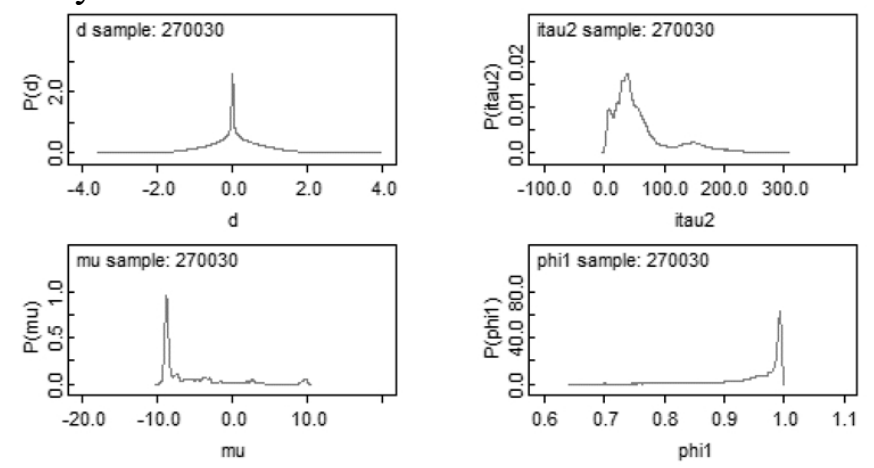

Fig. 4 The posterior distribution density function simulation 
From the table, we can obtain the Bayesian estimate of the parameter $\mu$ of the fluctuation level of the Shanghai and Shenzhen index based on the SV-MN model is -6.349; The fluctuating continuity parameter $\varphi$ of the Shanghai-Shenzhen index is 0.9626 , and the corresponding posterior confidence interval is [0.82, 0.9967]. It can be seen that the Shanghai-Shenzhen index has strong volatility persistence, and also shows that the stock market in our country has strong volatility persistence. From the table we can obtain the Bayesian estimate of the accuracy parameter $\tau$ of the Shanghai and Shenzhen index model is 58.81. Therefore, it can be inffered that under the SV-MN model, there is a great deal of noise in the Shanghai and Shenzhen stock markets. The Bayesian estimate of the parameter $d$ of the Shanghai-Shenzhen index in the table is 0.05861, indicating that there is a weak correlation between the Shanghai stock market's yield and the volatility.

\section{Analysis of fluctuations}

Through the analysis of the volatility of the stock market, we can know that China's stock market volatility has the characteristics of continuity, timeliness, agglomeration and so on, and there is a large amount of noise. The composition of the market will affect the volatility of the stock market to a large extent. Therefore, we analyze the causes of fluctuations from various perspectives such as investor composition, policy influence, investment psychology in the investment process, and manipulation behavior.

The Influence of Investor's Quality on Volatility. In the efficient market hypothesis, everyone on the market is a rational economic man. However, in real life, investors are not completely rational, and their purchase is based on both "noise" and information. These all have a direct impact on investors' decisions and ultimately reflect the market's prices. Therefore, the maturity of investors is very important for the stability of the securities market. We analyze the three aspects of investor structure, education and occupational status.

1) The investor structure analysis

From the table below, it can be seen that the number of individual investors has a significant growth trend. However, the number of institutional investors is very small. This reflects that there are a large number of noise traders in China's securities market. Regardless of information acquisition or utilization, institutional investors' professionalism is much higher than that of individual investors. This is one of the factors that leads to instability in the securities market.

\begin{tabular}{lrrrrrrrr}
\multicolumn{1}{l}{ INVESTORS (Year-end Figures) } & & & & & & 单位:万, 10000 \\
\hline & 2009 & 2010 & 2011 & 2012 & 2013 & 2014 & 2015 & 2016 \\
\hline No. of Personal Investors & 8542.60 & 9402.68 & 10091.18 & 10537.82 & 11047.50 & 11995.29 & 16899.70 & 20841.00 \\
$\quad$ No. of Company Investors & 28.15 & 30.88 & 33.40 & 35.48 & 38.06 & 41.58 & 49.22 & 56.12 \\
$\quad$ Total & 8570.75 & 9433.56 & 10124.57 & 10573.30 & 11065.56 & 12036.87 & 16858.92 & 20897.13 \\
\hline
\end{tabular}

Fig. 5 Investor Structure

2) Investor qualifications

Investor qualifications indirectly reflect the investor's knowledge reserves and the ability to analyze the status of the stock market. If the investor's academic qualification is higher, the ability to analyze the macroeconomics and individual stocks' fundamentals will be more correct, and the closer their investors' decisions are to the more rational.

TABLE 3 CONSTITUTION OF CHINESE INVESTORS IN 2017

\begin{tabular}{|l|l|l|l|}
\hline Education & Proportion & Education & Proportion \\
\hline Primary school and below & $0.25 \%$ & $\begin{array}{l}\text { Secondary/High School/Higher } \\
\text { Vocational School }\end{array}$ & $23.78 \%$ \\
\hline junior high school & $6.21 \%$ & Graduate student and above & $3.20 \%$ \\
\hline Bachelor/Specialist & $66.56 \%$ & & \\
\hline
\end{tabular}

From the table, it can be seen that most of the qualifications for stockholders in China are above college education, and academic qualifications are at a relatively high level. At the same time, only $3.20 \%$ of the graduates and above accounted for the fact that this country also lacks highly educated professionals.

3) Investor occupation status 
Investors engaged in finance-related occupations will have richer professional knowledge and complete knowledge framework than other professional investors, which will help him make more rational decisions, which will also reduce the night market fluctuations in the stock market.

TABLE 4 CHINESE INVESTOR OCCUPATION IN 2017

\begin{tabular}{|l|l|l|l|}
\hline Profession & Proportion & Profession & Proportion \\
\hline School student & $0.97 \%$ & Computers workers & $5.95 \%$ \\
\hline $\begin{array}{l}\text { Personnel / Administration / Senior } \\
\text { Management }\end{array}$ & $1.56 \%$ & $\begin{array}{l}\text { Education / Medical / Style } \\
\text { workers }\end{array}$ & $5.76 \%$ \\
\hline General Staff & $28.98 \%$ & Freelancers & $19.49 \%$ \\
\hline $\begin{array}{l}\text { Finance/Insurance/Banking } \\
\text { workers }\end{array}$ & $8.06 \%$ & Retired people & $4.01 \%$ \\
\hline Senior Management & $0.48 \%$ & Laid-off workers & $4.65 \%$ \\
\hline Civil servants & $4.96 \%$ & Others & $2.70 \%$ \\
\hline Business owner/operators & $3.23 \%$ & & \\
\hline
\end{tabular}

From the above table, we can see that the number of individual investors in the Chinese stock market engaged in financial services is relatively small, and non-financial investors mostly lack a complete knowledge structure. This is because China's senior high school education basically does not design personal finance courses, investors lack systematic learning, so they are more likely to rely on the suggestions of others and make irrational investments.

Impact of policies on volatility. In order to better reflect the relationship between volatility and stock market volatility, we compare the 2015-2016 policy with the stock market volatility index.

TABLE 5 MAJOR EVENTS IN CHINA'S STOCK MARKET

\begin{tabular}{|l|l|}
\hline Date & Events \\
\hline 2015.1 .16 & Two financial thresholds to return to 500,000 yuan \\
\hline 2015.5 .6 & The Umbrella Trust Umbrella has been newly opened and the parachute is still available \\
\hline 2015.5 .25 & Some brokers suspended the purchase of non-institutional umbrella accounts \\
\hline 2015.8 .21 & CITIC Securities caught up in the investigation of executives \\
\hline 2015.9 .15 & $\begin{array}{l}\text { Confirmation of the slowdown in the allocation of funds, and the replacement of the PB } \\
\text { system with securities companies can survive }\end{array}$ \\
\hline 2015.10 .19 & "Personal Account Type" Funding Comeback \\
\hline 2015.11 .25 & Regulatory window called stop-loss exchange swaps \\
\hline 2015.12 .30 & Merchants Shekou listed \\
\hline 2016.1 .1 & China's stock market implements a fuse mechanism \\
\hline 2016.2 .20 & Commission replaced the chairman \\
\hline 2016.3 .4 & China's stock market implements IPO registration \\
\hline 2016.6 .15 & Chinese stock market missed MSCI \\
\hline 2016.12 .1 & RMB joins SDR \\
\hline
\end{tabular}




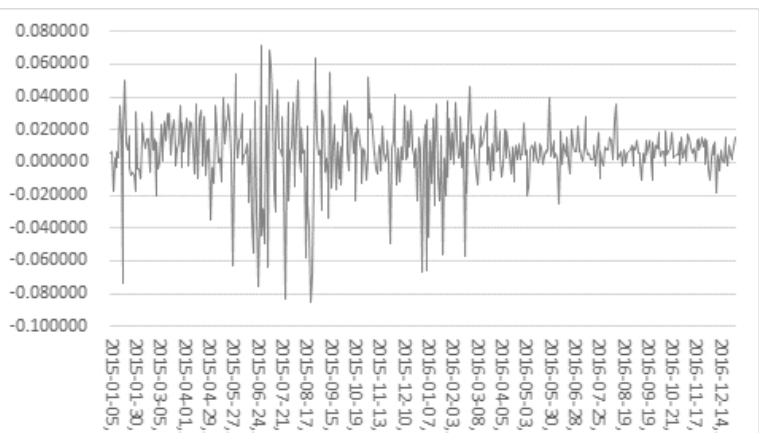

Fig. 6 2015-2016 China's stock market volatility chart

As can be seen from Fig.6, there are large fluctuations in most major policy points and fluctuations are frequent in two years. Unlike foreign stock markets, China's special economic system allows the government to exercise a certain level of intervention in the stock market. Such interventions will often disrupt the natural law of the market and thus affect market volatility. Through data analysis and finding relevant documents, we believe that the current policy interventions mainly have the following two problems:

4) The scope of policy intervention is too large. The government's intervention is almost everywhere in the trading market. The flow of funds and allocation of resources in the stock market depends first of all on the will of the government, not on the free choice of companies and investors.

5) Policy interventions are too frequent. As can be seen from the chart, a large part of the fluctuations are matched with the implementation time of the policy, indicating that a large part of the fluctuations originate from the influence of the policy. When the stock market is in a downturn and the stock price continues to fall, the government usually implements certain policies to achieve the goal of "supporting the market". However, when the stock market appears excessive speculation, when the stock price continues to rise, the government will create the opposite public opinion or policy to suppress the market. This will keep the stock market highly sensitive and alert to policy interventions. The pressure on the stock market's policies will continue to accumulate with the increase in the speculative time of the stock market, leading to huge volatility in the stock price when the policy effect disappears.

The Influence of Investor's Sentiment on Volatility. Investor sentiment is an important factor reflecting investor psychology. When the market price signal is received, the investor's sentiment will be affected. At the same time, investor sentiment will have a major impact on investor behavior and decision making, which will affect the returns and volatility of the stock market. However, since the overall quality of individual investors in China is not high, their mood is easily affected by institutional investors and other market information. This will lead to the continued spread and expansion of market noise, which will easily form a herd effect and cause large fluctuations in the Chinese stock market.

\section{Analysis}

The volatility of China's stock market returns is very complex. It contains information on market changes and investment risks. Therefore, studying the characteristics of China's stock market volatility and influencing factors has important theoretical and practical significance for the stock This article collects closing prices of the Shanghai and Shenzhen closing index from January 4, 2010 to December 29, 2017, and simulates the volatility of the stock market by using the MCMC algorithm to simulate the parameters of the SV-MN model. We can get the characteristics of sustainability, timeliness, and agglomeration of the stock market volatility in China, and there is a large amount of noise. Then we analyze the causes of the fluctuations from various perspectives such as the structure of investors, related policies, the investment psychology in the investment process, and speculative manipulation. Based on the empirical test of the empirical model, this paper proposes relevant suggestions for promoting stable and healthy development of the stock market in China: Since the stock market is heavily influenced by national policies, financial services industry must pay attention 
to the indirect effects of policies on the market and adjust the management strategies in a timely manner. In addition, the financial services industry should pay attention to the impact of noise traders on market fluctuations, strengthen the rational education of investors, increase the investment background of retail investors, strengthen financial supervision and strictly combat speculation and manipulation.

\section{References}

[1]Shen Yue, Zhang Cheng. Research on Volatility of China's Agricultural Product Futures Market Based on MCMC Algorithm[J]. Journal of Northwest A\&F University(Social Science Edition), 2016, 16(6):114-120.

[2]Mao Minglai, Chen Tong, Xu Zhengguo. Discussion on SV Model System[J]. Journal of Northwest A\&F University(Social Science Edition), 2006, 6(4):65-68.

[3]Meng Lifeng, Zhang Shiying, He Xin. Bayesian Analysis of SV Model with Leverage Effect and Its Application[J]. Systems Engineering, 2004, 22(3):47-51.

[4]Li Xiaoming. SV model selection based on Bayesian factors[D]. Zhejiang University, 2011.

[5]Gao Daliang, Zhang Xiaoyong. Investor Sentiment and Stock Market Volatility Decomposition[J]. Journal of Hunan University(Social Science), 2013, 27(5):62-66.

[6]Zhang Weihua. Research on stock market asymmetry correlation based on SV model[D]. Changchun University of Technology, 2016.

[7]Li Feng. Analysis of Bayesian Financial Stochastic Volatility Model Based on MCMC Simulation[D]. Hunan University, 2007.

[8]Zhao Huiqin, Liu Jinshan. A Stochastic Volatility Model of Shanghai Stock Market Using MCMC Method[J]. Journal of Huaqiao University(Natural Science), 2017, 38(2): 262-265.

[9]Liu Shuqi. Research on ValR Estimation of Stock Market Returns Based on MCMC-GARCH Model[D]. Harbin Institute of Technology, 2015. 